\title{
Higher Genus Superstring Amplitudes From the Geometry of Moduli Space
}

\author{
Marco Matone and Roberto Volpato \\ Dipartimento di Fisica "G. Galilei”, Istituto Nazionale di Fisica Nucleare, \\ Università di Padova, Via Marzolo, 8 - 35131 Padova, Italy
}

\begin{abstract}
We show that the higher genus 4-point superstring amplitude is strongly constrained by the geometry of moduli space of Riemann surfaces. A detailed analysis leads to a natural proposal which satisfies several conditions. The result is based on the recently derived Siegel induced metric on the moduli space of Riemann surfaces and on combinatorial products of determinants of holomorphic abelian differentials.
\end{abstract}

June 2005 


\section{Introduction}

One basic problem in superstring theory concerns the explicit evaluation of the higher genus amplitudes. A fundamental step in such a direction has been done by D'Hoker and Phong that in a series of remarkable papers [1] [2] [3], provided an explicit gauge slice independent formulation of the NSR superstring at genus two. Their analysis also provides relevant suggestions for the higher genus generalization. The fact that the quantum string is described in terms of classical geometry of the moduli space suggests trying to investigate the structure of the higher genus amplitudes from purely algebraic-geometrical considerations.

In recent years, a new covariant formulation of the superstring has been proposed by Berkovits [4[-16]. In this respect we observe that the analysis of (super)strings leads to consider basic properties of the moduli space of Riemann surfaces. Below we will comment on a possible algebraic-geometrical interpretation of the pure spinor condition. Such a condition is a bilinear relation on differentials, whose weight is changed after the twisting, depending on the superstring critical dimension. Known classical relations among differentials concern, e.g., the characterization of a basis of holomorphic quadratic differentials, out of the $g(g+1) / 2$ one may get by bilinear combinations of the holomorphic one-differentials (Schottky problem). Other relations among holomorphic differentials which, like the pure spinor condition, involves the critical dimension, is provided by the Mumford isomorphism.

In this paper we focus on the higher genus 4-point superstring amplitude. In the case of genus 2, this problem has been first considered by Iengo and Zhu [17]. Following the methods of [1], an explicit formula, still gauge slice dependent, has been derived in [18]. The basic problem of a fully gauge slice independent formulation has been finally resolved in [2]

$$
A_{4}^{2-\text { loop }}=B_{2} \int_{\mathcal{M}_{2}} \frac{\left|\bigwedge_{i \leq j}^{2} \mathrm{~d} \Omega_{i j}\right|^{2}}{(\operatorname{det} \operatorname{Im} \Omega)^{3}} \int_{\Sigma^{4}} \frac{\left|\mathcal{Y}_{S}\right|^{2}}{(\operatorname{det} \operatorname{Im} \Omega)^{2}} e^{-\sum_{i<j} k_{i} \cdot k_{j} G\left(z_{i}, z_{j}\right)},
$$

where

$$
\begin{gathered}
\mathcal{Y}_{S}=\left(k_{1}-k_{2}\right) \cdot\left(k_{3}-k_{4}\right) \Delta\left(z_{1}, z_{2}\right) \Delta\left(z_{3}, z_{4}\right)+2 \leftrightarrow 3+2 \leftrightarrow 4, \\
G(z, w):=-\ln |E(z, w)|^{2}+2 \pi \operatorname{Im} \int_{z}^{w} \omega_{i}(\operatorname{Im} \Omega)_{i j}^{-1} \operatorname{Im} \int_{z}^{w} \omega_{j},
\end{gathered}
$$

and

$$
\Delta(z, w):=\omega_{1}(z) \omega_{2}(w)-\omega_{2}(z) \omega_{1}(w),
$$

is the basic holomorphic antisymmetric bi-differential which plays the role of building block for the genus 2 superstring amplitudes. A preliminary investigation concerning the extension to higher loop of (1.1) has been considered in [19]. A similar formula has been recently derived by Berkovits in his pure spinor formalism [⿴囗⿴囗十). 
To find the higher genus extension of such an amplitude requires considering two problems

1. Find the higher genus version of the modular invariant measure

$$
\frac{\left|\bigwedge_{i \leq j}^{2} \mathrm{~d} \Omega_{i j}\right|^{2}}{(\operatorname{det} \operatorname{Im} \Omega)^{3}}
$$

Whereas in the case of genus 3 the analog of such a volume form can still be used, except for the hyperelliptic locus where by Nöther theorem the bilinears $\omega_{i}(z) \omega_{j}(z)$ span a $(2 g-1)$-dimensional subspace of $H^{0}\left(\Sigma, K^{2}\right)$, in the case $g>3$ the $\frac{1}{2} g(g+1)$ dimensional space of symmetric $g \times g$ matrix with positive definite imaginary part, should satisfy conditions leading to a subspace parametrizing the moduli space.

2. The other problem with the higher genus extension of 4-point amplitude concerns the generalization of

$$
\int_{\Sigma^{4}} \frac{\left|\mathcal{Y}_{S}\right|^{2}}{(\operatorname{det} \operatorname{Im} \Omega)^{2}} e^{-\sum_{i<j} k_{i} \cdot k_{j} G\left(z_{i}, z_{j}\right)} .
$$

On the other hand, whereas $G\left(z_{i}, z_{j}\right)$ is canonically defined for any $g$, there is not an obvious extension of $\mathcal{Y}_{S}$. However, we will see that we are essentially forced to still use the determinants of the holomorphic one-differentials as building block for constructing the $\mathcal{Y}_{S}$. This implies that one has to introduce $g-2$ more points for each determinant. Since the determinants come in pair, we need to introduce $2 g-4$ points. We then are lead to analyze the structure of the possible permutations between such points and then integrating on $\Sigma^{2 g-4}$.

Then our proposal is

$$
A_{4}^{g-\text { loop }}=B_{g} \int_{\mathcal{M}_{g}}|\operatorname{det} g|^{1 / 2} \mathrm{~d} \Xi_{1} \wedge \ldots \wedge \mathrm{d} \bar{\Xi}_{3 g-3} \mathcal{F}_{g}\left(k_{i}\right),
$$

where $g$ is the Siegel induced metric derived in [20], $\mathrm{d} \Xi_{i}, i=1, \ldots, 3 g-3$ are the moduli induced by the constraints on $d \Omega_{i j}$ [20]. Furthermore,

$$
\mathcal{F}_{g}\left(k_{i}\right)=\int_{\Sigma^{2 g}} \frac{\left|\mathcal{Y}_{S}\right|^{2}}{(\operatorname{det} \operatorname{Im} \Omega)^{2}} e^{-\sum_{i<j} k_{i} \cdot k_{j} G\left(z_{i}, z_{j}\right)},
$$

and

$$
\mathcal{Y}_{S}=\left(k_{1}-k_{2}\right) \cdot\left(k_{3}-k_{4}\right) \mathcal{H}_{12,34}+2 \leftrightarrow 3+2 \leftrightarrow 4
$$

where $\mathcal{H}_{12,34}$ is a sum on a set of permutations of $2 g-4$ points of terms such as

$$
X_{12}\left(w_{1 \rightarrow g-2}\right) X_{34}\left(w_{g-1 \rightarrow 2 g-4}\right),
$$


where

$$
X_{i j}\left(w_{k \rightarrow m}\right):=\operatorname{det} \omega\left(z_{i}, z_{j}, w_{k}, \ldots, w_{m}\right) .
$$

Defining $\mathcal{Y}_{S}$ in terms of determinants of holomorphic differentials and then integrating on the $2 g-4$ variables $\left\{w_{k}\right\}$, provides a way to get modular invariant linear combinations, of terms such as $\left|\Delta_{i j}\left(z_{1}, z_{2}\right) \Delta_{k l}\left(z_{3}, z_{4}\right)\right|^{2}$, where $\Delta_{i j}\left(z_{k}, z_{l}\right):=\omega_{i}\left(z_{k}\right) \omega_{j}\left(z_{l}\right)-\omega_{j}\left(z_{k}\right) \omega_{i}\left(z_{l}\right)$, with the coefficients given by bilinears in the minors of order $g-2$ of $\operatorname{Im} \Omega$ divided by $(\operatorname{det} \operatorname{Im} \Omega)^{2}$. For example, as we will see, integrating over $w_{1}, \ldots, w_{g-2}$ the simplest product, we have

$$
\begin{gathered}
\frac{1}{2^{g-2}(g-2) !} \int_{\Sigma^{g-2}} \prod_{k}\left|d w_{k}\right|^{2} \operatorname{det} \omega\left(z_{1}, z_{2}, w_{1}, \ldots, w_{g-2}\right) \operatorname{det} \bar{\omega}\left(z_{3}, z_{4}, w_{1}, \ldots, w_{g-2}\right)= \\
\sum_{\substack{i<j \\
m<n}}(-1)^{i+j+m+n} \Delta_{i j}\left(z_{1}, z_{2}\right) \bar{\Delta}_{m n}\left(z_{3}, z_{4}\right) \operatorname{det}_{\substack{k \neq i, j \\
l \neq m, n}} \operatorname{Im} \Omega_{k l} .
\end{gathered}
$$

From a geometrical point of view, our investigation corresponds to find the higher genus analog of (1.1). Thus, besides modular invariance, one should care about the absence of ambiguities, such as the dependence on the choice of arbitrary points in the higher loop generalization of (1.1). Using the determinants of the standard basis of the holomorphic differentials and then integrating over the additional $2 g-4$ points removes possible ambiguities and guarantees modular invariance. Therefore, the main properties of the amplitude we derived are
a. Manifestly ambiguity free.
b. Modular invariance.
c. Reproduces the $g=2$ expression.

Among the possible permutations of the points $\left\{w_{k}\right\}$, we will focus on two particularly simple cases. The simplest one corresponds to consider

$$
\mathcal{H}_{12,34}=X_{12}\left(w_{1 \rightarrow g-2}\right) X_{34}\left(w_{g-1 \rightarrow 2 g-4}\right)+X_{34}\left(w_{1 \rightarrow g-2}\right) X_{12}\left(w_{g-1 \rightarrow 2 g-4}\right)
$$

so that (1.3) guarantees the invariance of $\mathcal{Y}_{S}$ under the simultaneous exchange of $k_{i} \leftrightarrow k_{j}$, $z_{i} \leftrightarrow z_{j}$. Since this is also the property of the exponential in (1.2), the resulting amplitude is symmetric under exchange of the momenta.

It is interesting to understand the general structure of $\mathcal{H}_{i j, k l}$ once one considers different choices of the possible permutations, an issue considered in detail in the paper. Since by construction the amplitude is invariant under permutation of the external momenta, it is clear that different choices of permutations of the $\left\{w_{k}\right\}$ will always lead to an expression of $\int_{\Sigma^{2 g}}\left|\mathcal{Y}_{S}\right|^{2}$ proportional to $\left(s^{2}+t^{2}+u^{2}\right)(\operatorname{det} \operatorname{Im} \Omega)^{2}$, where the term $(\operatorname{det} \operatorname{Im} \Omega)^{2}$ follows 
by the properties of $\left|\mathcal{Y}_{S}\right|^{2}$ under modular transformations. Nevertheless, because of the exponential of the Green-functions, different choices would in principle lead to different expressions for the amplitude.

The fact that modular invariance essentially leads to introduce determinants of the canonical basis of holomorphic one-differentials differentials $\omega_{k}$, suggests that the pair of extra $g-2$ differentials $\omega_{k}$ is due to zero modes. In the standard approach such zero modes are related to the spin structures of the $\beta-\gamma$ system, while, interestingly enough, in the Berkovits approach the zero modes are related to other elementary fields.

We note that in [4], besides the derivation of the 4-point $g=2$ superstring amplitude, Berkovits also notes that preliminary calculations in his formulation indicate that the momentum dependence of the higher genus 4-point amplitude is the same of the one in genus two, in agreement with the suggestion by Zhu in [19]. On the other hand, on general grounds, it is difficult to understand the mechanism for which terms giving contributions to $D^{4} R^{4}$, having the same analytic general structure of the genus two case, may be suppressed. However, such a possibility cannot be excluded, for example by summing on antisymmetric permutations of the $\left\{w_{k}\right\}$, an operation which is reminiscent of the summation over the odd spin structures, one may get a vanishing result. Nevertheless it remains the problem of finding an analytic structure for the amplitude which takes place only at genus greater than two. Another possibility is that for $g>2$ the hyperelliptic contribution, that we do not consider here, may lead to some cancellation mechanism. On the other hand, the inclusion of the hyperelliptic locus corresponds to a generalization of (1.2) involving essentially only the measure. Since the hyperelliptic locus has codimension $g-2$, also in this case one should understand the structure of such a cancellation. It would be interesting to fully understand the basic role of $S$-duality for the higher genus amplitudes along the lines investigated in [21].

The paper is organized as follows. In section 2 we first consider the problems which arise in choosing additional points in defining the $g \times g$ matrices $\omega_{i}\left(x_{j}\right)$. We will also comment on some properties satisfied by the basis of holomorphic differentials which are related both to the problem of the measure on $\mathcal{M}_{g}$ (Schottky problem), and to some speculation on the possible geometrical understanding of the pure spinor condition. Next, we will shortly review the measure on the (coarse) moduli space corresponding to the generalization to higher genus of the $g=2$ measure.

In section 3 we will start by constructing $\mathcal{Y}_{S}$ by means of a suitable combination of products of determinants. We then will consider in some detail the underlying combinatorial structure which arises in considering the permutations of the additional points $\left\{w_{k}\right\}$. As we will see, the kind of analysis suggests further developments which may be of mathematical interest as well.

Finally, section 4 is devoted to conclusions and suggestions for future investigations. 


\section{Geometry of $\mathcal{M}_{g}$ and the modular invariant metric}

There are two main questions in considering the higher genus generalization of the 4particle amplitude, the measure on the moduli space and the higher genus generalization of the holomorphic bi-differential $\Delta(z, w)$. Let us first concentrate on the latter. On general grounds, we should look at the simplest possible generalization. This would suggest that even in higher genus

$$
\Delta(z, w)=\operatorname{det} \omega_{i}\left(z_{j}\right)
$$

with $z_{1}=z$ and $z_{2}=w$. However, such an expression would require choosing the $g-2$ points $z_{i}, i=3, \ldots, g$, that, in order to avoid ambiguities in defining the amplitude, should be chosen in a canonical way. Also note that a naive integration along cycles of $\Sigma$ on each of the $g-2$ points would spoil the modular properties of $\Delta\left(z_{i}, z_{j}\right)$. At first sight, however, there is no a canonical way to fix such a set of points. In particular, we cannot expect that this set would correspond to the zeroes of some differential. The reason is that the degree of a $\lambda$-differential is $2 \lambda(g-1)$, so that fixing a set of canonical points should require a number of points multiple of $g-1$. A typical example concerns the $g-1$ zeroes of the holomorphic one-differentials constructed in terms of the $\theta$-function with odd spin structure. This zero set depends on the period matrix and on the particular odd spin structure, so it reduces the freedom in choosing the points to the choice of the particular odd spin structure.

There are several structures that start emerging at genus three. One concerns the dimensionality of the locus where the $\theta$-function vanishes identically. To understand when this happens, note that linear independence of the $g$ holomorphic abelian differentials may fail at specific points. This means that there are divisors for which the rows of $\omega_{i}\left(z_{j}\right)$ are linearly dependent. Writing $\operatorname{det} \omega_{i}\left(z_{j}\right)$ in terms of $\theta$-functions and prime forms, one sees that such a locus of super zeroes is $g-2$ dimensional.

Another place where appears a dependence on $g-2$, concerns the systematic construction by Petri of a basis for $H^{0}\left(\Sigma, K^{2}\right)$ by means of bilinears of a suitable basis of $H^{0}(\Sigma, K)$ [22] (see also [20] and 23] for related constructions). In turn, this is related to the mentioned fact that the codimension of the hyperelliptic locus in the moduli space of Riemann surfaces is just $g-2$.

\subsection{Critical dimension and Mumford isomorphism.}

The geometry of the moduli space is deeply connected with the field-theoretical formulation of string theory. In particular, there are properties, such as the critical dimension, which are directly connected to basic relations satisfied by the holomorphic differentials. For example, in the $g>3$ non-hyperelliptic case, constructing a basis of holomorphic

1 For hyperelliptic Riemann surfaces of $g=3$ one can use a $\theta$-function with even characteristic. 
quadratic differentials in terms of bilinear combinations of the canonical basis of holomorphic one-differentials $\omega_{i}$ is the Schottky problem. A twisting in the field content may be connected to such relations. There is another relevant relationship between holomorphic one- and two-differentials, the Mumford isomorphism

$$
K \simeq E^{13},
$$

where $E$ is the Hodge line bundle and $K$ the determinant line bundle. Schottky problem and Mumford isomorphism involve strictly related structures. On the other hand, the Mumford isomorphism provides an algebraic-geometrical understanding of the bosonic critical dimension [23] 24]. We also note that such issues are strictly related to the problem of classifying the higher genus modular forms, and so to the problem of writing the Mumford form in terms of $\theta$-constants and related objects (see e.g. [25]).

There is another relationship involving differentials on Riemann surfaces and critical dimension. This is the pure spinor condition

$$
\lambda_{\alpha} \gamma_{\alpha \beta}^{m} \lambda_{\beta}=0, \quad \bar{\lambda}_{\alpha} \gamma_{\alpha \beta}^{m} \bar{\lambda}_{\beta}=0 .
$$

Here $m$ runs from 0 to 9 and $\gamma_{\alpha \beta}^{m}$ are $16 \times 16$ matrices which are off-diagonal blocks of the $32 \times 32$ ten-dimensional $\Gamma$-matrices and satisfy

$$
\gamma_{\alpha \beta}^{m} \gamma^{n \beta \gamma}=2 \eta^{m n} \delta_{\alpha}^{\gamma} .
$$

Originally the $\lambda$ 's and $\bar{\lambda}$ 's are considered $\left(\frac{1}{2}, 0\right)$-differentials and $\left(0, \frac{1}{2}\right)$-differentials respectively. After twisting the $\lambda$ 's become $(0,0)$-differentials and $\bar{\lambda}$ 's $(0,1)$-differentials.

Note that bilinear relations among holomorphic abelian differentials may lead to conditions on scalars and 1/2-differentials. This suggests that the pure spinor condition (2.2) may be related to the Schottky problem. In this respect, it is worth noticing that the Schottky problem itself is related to the uniformization problem via the Liouville equation [26].

Let us also notice that in the pure spinor formalism the $b$-ghost is a composite field. It would be interesting to understand whether there exists a suitable combination of zero modes of elementary fields that may suitably combine to build the volume form on $\mathcal{M}_{g}$ made of quadratic holomorphic differentials.

\subsection{The induced measure}

We now consider the problem of defining the higher genus version of the measure on the moduli space in (1.1). Let $\hat{\mathcal{M}}_{g}$ be the locus of moduli space of compact nonhyperelliptic Riemann surfaces of genus $g \geq 4$. The restriction on $\hat{\mathcal{M}}_{g}$ of the measure on 
the Siegel upper half-space has been derived in [20]. In the following we briefly review the results of this paper.

Let $\Sigma$ be a compact non-hyperelliptic Riemann surface of genus $g \geq 4$ and $\left\{\omega_{1}, \ldots, \omega_{g}\right\}$ the canonical basis of $H^{0}(\Sigma, K)$, with $K$ the canonical line bundle of $\Sigma$. Let $p_{1}, \ldots, p_{g}$ be a set of points in $\Sigma$ such that

$$
\operatorname{det} \omega\left(p_{1}, \ldots, p_{g}\right) \neq 0
$$

where $\operatorname{det} \omega\left(p_{1}, \ldots, p_{g}\right):=\operatorname{det} \omega_{i}\left(p_{j}\right)$. Then

$$
\sigma_{i}(z):=\frac{\operatorname{det} \omega\left(p_{1}, p_{2}, \ldots, p_{i-1}, z, p_{i+1}, \ldots, p_{g}\right)}{\operatorname{det} \omega\left(p_{1}, \ldots, p_{g}\right)}
$$

$i=1, \ldots, g$, is a basis of $H^{0}(\Sigma, K)$.

We introduce the $g(g+1) / 2$-dimensional vector $v$

$$
v_{k}:= \begin{cases}\sigma_{k}^{2}, & k=1, \ldots, g, \\ \sigma_{i+j} \sigma_{j}, & k=i+j(2 g-j+1) / 2,\end{cases}
$$

where $j=1, \ldots, g-1, i=1, \ldots, g-j$. If we assume that the divisors of $\left(\sigma_{i}\right)$ consist of distinct points, then $\left\{v_{j}\right\}:=\left\{v_{1}, \ldots, v_{3 g-3}\right\}$ is a modular invariant basis of $H^{0}\left(\Sigma, K^{2}\right)$, the space of the holomorphic quadratic differentials on $\Sigma$.

Let $W(P)$ be the Wronskian $W\left(v_{1}, \ldots, v_{3 g-3}\right)(P)$ of the basis $\left\{v_{j}\right\}$ at a generic point $P \in \Sigma$ and $\hat{W}_{k, i j}(P)$ be the Wronskian $W\left(v_{1}, \ldots, v_{k-1}, \omega_{i} \omega_{j}, v_{k+1}, \ldots, v_{3 g-3}\right)(P)$ at $P$. We have

\section{Theorem 1.}

$$
\omega_{i}(z) \omega_{j}(z)=\sum_{k=1}^{3 g-3} \frac{\hat{W}_{k, i j}}{W} v_{k}(z)
$$

where the ratio $\hat{W}_{k, i j} / W$ does not depend on the point $P$.

The line element $d s$ on the Siegel upper half-space

$$
d s^{2}:=\operatorname{Tr}\left[(\operatorname{Im} \Omega)^{-1} d \Omega(\operatorname{Im} \Omega)^{-1} d \bar{\Omega}\right]
$$

defines the volume element

$$
\frac{\left|\bigwedge_{i \leq j}^{g} \mathrm{~d} \Omega_{i j}\right|^{2}}{(\operatorname{det} \operatorname{Im} \Omega)^{g+1}}
$$


Let $k$ be the Kodaira-Spencer map identifying the quadratic differentials on $\Sigma$ with the fiber of the cotangent of the Teichmüller space at the point representing $\Sigma$. We have

$$
k\left(\omega_{i} \omega_{j}\right)=(2 \pi i)^{-1} d \Omega_{i j} .
$$

Let us set

$$
d \Xi_{i}:=2 \pi i k\left(v_{i}\right), \quad i=1, \ldots, 3 g-3 .
$$

By Eq. (2.4) we have

$$
d \Omega_{i j}=\sum_{k=1}^{3 g-3} \frac{\hat{W}_{k, i j}}{W} d \Xi_{k}
$$

$i, j=1, \ldots, g$.

Theorem 2. The line element $d s_{\mid \hat{\mathcal{M}}_{g}}$ on $\hat{\mathcal{M}}_{g}$ induced by the Siegel metric is

$$
d s_{\mid \hat{\mathcal{M}}_{g}}^{2}:=\sum_{i, j=1}^{3 g-3} g_{i j} d \Xi_{i} d \bar{\Xi}_{j},
$$

where

$$
g_{i j}:=|W|^{-2} \operatorname{Tr}\left[(\operatorname{Im} \Omega)^{-1} \hat{W}_{i}(\operatorname{Im} \Omega)^{-1} \hat{W}_{j}\right] .
$$

It follows that the Siegel induced modular invariant volume form on $\hat{\mathcal{M}}_{g}$ is

$$
d \nu:=|\operatorname{det} g|^{\frac{1}{2}} d \Xi_{1} \wedge \ldots \wedge d \bar{\Xi}_{3 g-3} .
$$

\section{The modular function $\mathcal{Y}_{S}$.}

In the following we will define the higher genus version of the genus 2 formula for $\mathcal{Y}_{S}$. In particular, we generalize the building blocks $\Delta\left(z_{1}, z_{2}\right) \Delta\left(z_{3}, z_{4}\right)$, used to construct $\mathcal{Y}_{S}$ in the genus two case, as a product of a pair of determinants of holomorphic one-differentials on the Riemann surface $\Sigma$. We will be forced to introduce $2 g-4$ arbitrary points, that will be integrated over $\Sigma^{2 g-4}$ in order to obtain an unambiguous result. The formula will result to be modular invariant and to reproduce the genus 2 expression of the 4-point superstring amplitude.

At genus 3, the above requirements fix, up to a normalization constant, the form of $\mathcal{Y}_{S}$. At higher genus we have to deal with several possibilities. Although we will consider all these possibilities, we will show that there exists a simple formula which seems a natural generalization of the genus two case. 


\subsection{Definitions and properties}

Let us define

$$
\begin{array}{ll}
X\left(w_{k \rightarrow n}\right) & :=\operatorname{det} \omega\left(w_{k}, \ldots, w_{n}\right), \\
X_{i j}\left(w_{k \rightarrow m}\right) & :=\operatorname{det} \omega\left(z_{i}, z_{j}, w_{k}, \ldots, w_{m}\right), \\
X\left(w_{\rho(k \rightarrow n)}\right) & :=\operatorname{det} \omega\left(w_{\rho(k)}, \ldots, w_{\rho(n)}\right), \\
X_{i j}\left(w_{\rho(k \rightarrow m)}\right) & :=\operatorname{det} \omega\left(z_{i}, z_{j}, w_{\rho(k)}, \ldots, w_{\rho(m)}\right),
\end{array}
$$

for all $k, n, m \in \mathbb{N}$, with $n-k+1=g$ and $m-k+1=g-2$, and set

$$
\mathcal{H}_{i j, k l}:=\sum_{\rho \in B_{2 g-4}} C(\rho) X_{i j}\left(w_{\rho(1 \rightarrow g-2)}\right) X_{k l}\left(w_{\rho(g-1 \rightarrow 2 g-4)}\right)
$$

where $w_{1}, \ldots, w_{2 g-4}$ are points in $\Sigma, C(\rho)$ are coefficients in $\mathbb{C}$ and $B_{2 g-4}$ is a subset, that we will define below, of the group of permutations of $\{1,2, \ldots, 2 g-4\}$. Essentially, we want the elements in $B_{2 g-4}$ to be in one to one correspondence with all the different ways of choosing $g-2$ points out of the $2 g-4$ points $\left\{w_{i}\right\}$. Therefore, we require that for any pair $\rho, \sigma \in B_{2 g-4}, \rho \neq \sigma$ implies $\rho(\{1,2, \ldots, g-2\}) \neq \sigma(\{1,2, \ldots, g-2\})$. There is not a unique way to choose the elements in $B_{2 g-4}$; different choices can give some minus sign that can be reabsorbed by a redefinition of the coefficients $C(\rho)$.

We also impose the following constraint on $\{C\}$ : if a pair $\rho, \sigma \in B_{2 g-4}$, satisfies $\rho(\{1,2, \ldots, g-2\})=\sigma(\{g-1, g, \ldots, 2 g-4\})$ then $C(\rho)=C(\sigma)$. This ensures that $\mathcal{H}_{i j, k l}=\mathcal{H}_{k l, i j}$. One can verify that, at genus $g$, we have to fix $(2 g-4) ! / 2[(g-2) !]^{2}$ independent coefficients $C$. Each of them appears twice in (3.1). We can avoid this redundancy using the equivalent definition

$$
\begin{gathered}
\mathcal{H}_{i j, k l}:= \\
\left.\sum_{\rho \in B_{2 g-4}^{\prime}} C(\rho)\left[X_{i j}\left(w_{\rho(1 \rightarrow g-2)}\right) X_{k l}\left(w_{\rho(g-1 \rightarrow 2 g-4)}\right)+X_{k l}\left(w_{\rho(1 \rightarrow g-2)}\right) X_{i j}\left(w_{\rho(g-1 \rightarrow 2 g-4)}\right)\right)\right],
\end{gathered}
$$

where $\rho \in B_{2 g-4}^{\prime} \subset B_{2 g-4}$ satisfies the condition $\rho(1) \leq g-2$. We define

$$
\mathcal{Y}_{S}:=\left(k_{1}-k_{2}\right) \cdot\left(k_{3}-k_{4}\right) \mathcal{H}_{12,34}+2 \leftrightarrow 3+2 \leftrightarrow 4,
$$

and propose that the integral in $\Sigma^{4}$ is now replaced by the integral over $\Sigma^{2 g}$

$$
\mathcal{F}_{g}\left(k_{i}\right):=\int_{\Sigma^{2 g}} \frac{\left|\mathcal{Y}_{S}\right|^{2}}{(\operatorname{det} \operatorname{Im} \Omega)^{2}} e^{-\sum_{i<j} k_{i} \cdot k_{j} G\left(z_{i}, z_{j}\right)}
$$


where $G\left(z_{1}, z_{2}\right)=-\ln \left|E\left(z_{1}, z_{2}\right)\right|^{2}+2 \pi \operatorname{Im} \int_{z_{1}}^{z_{2}} \omega_{i}(\operatorname{Im} \Omega)_{i j}^{-1} \operatorname{Im} \int_{z_{1}}^{z_{2}} \omega_{j}$. Under a modular transformation $\tilde{\Omega}=(A \Omega+B) \cdot(C \Omega+D)^{-1}$, we have

$$
\begin{aligned}
\operatorname{det} \operatorname{Im} \tilde{\Omega} & =|\operatorname{det}(C \Omega+D)|^{-2} \operatorname{det} \operatorname{Im} \tilde{\Omega}, \\
\left|\tilde{\mathcal{Y}}_{S}\right|^{2} & =|\operatorname{det}(C \Omega+D)|^{-4}\left|\mathcal{Y}_{S}\right|^{2},
\end{aligned}
$$

so that $\mathcal{F}_{g}\left(k_{i}\right)$ is modular invariant.

Performing the integration over the $2 g-4$ variables $\left\{w_{k}\right\}$ in (3.2) results in a linear combination of terms such as $\left|\Delta_{i j}\left(z_{1}, z_{2}\right) \Delta_{k l}\left(z_{3}, z_{4}\right)\right|^{2}$, times $e^{-\sum_{i<j} k_{i} \cdot k_{j} G\left(z_{i}, z_{j}\right)}$, where

$$
\Delta_{i j}\left(z_{k}, z_{l}\right):=\omega_{i}\left(z_{k}\right) \omega_{j}\left(z_{l}\right)-\omega_{j}\left(z_{k}\right) \omega_{i}\left(z_{l}\right)
$$

with the coefficients given by bilinears in the minors of order $g-2$ of $\operatorname{Im} \Omega$ divided by $(\operatorname{det} \operatorname{Im} \Omega)^{2}$. To see this, first observe that since the determinant of a $n \times n$ matrix can be written as

$$
\operatorname{det} a=\epsilon_{i_{1} \ldots i_{n}} a_{1 i_{1}} \ldots a_{n i_{n}}=\frac{1}{n !} \epsilon_{i_{1} \ldots i_{n}} \epsilon_{j_{1} \ldots j_{n}} a_{i_{1} j_{1}} \ldots a_{i_{n} j_{n}}
$$

we have

$$
\operatorname{det} \int_{\Sigma}|d z|^{2} \omega_{i} \bar{\omega}_{j}=\frac{1}{g !} \int_{\Sigma^{g}} \prod_{k}\left|d z_{k}\right|^{2} \epsilon_{i_{1} \ldots i_{g}} \epsilon_{j_{1} \ldots j_{g}} \omega_{i_{1}}\left(z_{1}\right) \bar{\omega}_{j_{1}}\left(z_{1}\right) \ldots \omega_{i_{g}}\left(z_{g}\right) \bar{\omega}_{j_{g}}\left(z_{g}\right)
$$

so that, considering the index of the integration variables as matrix index,

$$
\frac{1}{g !} \int_{\Sigma^{g}} \prod_{k}\left|d z_{k}\right|^{2}\left|\operatorname{det} \omega_{i}\left(z_{j}\right)\right|^{2}=\operatorname{det} \int_{\Sigma} \omega_{i} \wedge \bar{\omega}_{j}=2^{g} \operatorname{det} \operatorname{Im} \Omega_{i j}
$$

where

$$
\int_{\Sigma}|d z|^{2} \omega_{i} \bar{\omega}_{j}:=-i \int_{\Sigma} \omega_{i} \wedge \bar{\omega}_{j}=2 \operatorname{Im} \Omega_{i j}
$$

In order to explicitly perform the integration of the $2 g-4$ variables $\left\{w_{i}\right\}$ in (3.2), we factorize the dependence on the $\left\{z_{i}\right\}$ in $\mathcal{H}_{i j, k l}$. To this end, note that

$$
\epsilon_{i_{1} \ldots i_{g}}=(-1)^{i_{1}+i_{2}+1} \epsilon_{i_{1} i_{2}} \epsilon_{i_{3} \ldots i_{g}}^{\left(i_{1} i_{2}\right)}
$$

where the value of the superscripts $i_{1}$ and $i_{2}$ are excluded from the range of values of $i_{k>2}$ and $\epsilon_{i_{1} i_{2}}=1, \epsilon_{i_{3} \ldots i_{g}}^{\left(i_{1} i_{2}\right)}=1$ if $i_{1}<i_{2}$ and $i_{3}<\ldots<i_{g}$, respectively. Therefore, we have

$$
\operatorname{det} \omega\left(z_{1}, z_{2}, w_{1}, \ldots, w_{g-2}\right)=\sum_{i<j}(-1)^{i+j+1}\left[\omega_{i}\left(z_{1}\right) \omega_{j}\left(z_{2}\right)-\omega_{j}\left(z_{1}\right) \omega_{i}\left(z_{2}\right)\right] \operatorname{det}_{k \neq i, j} \omega_{k}\left(w_{l}\right),
$$


where $l=1, \ldots, g-2$. By means of such a factorization, we may perform the integration on the $2 g-4$ variables $\left\{w_{i}\right\}$ in (3.2). For example, by (3.5) and (a version of) (3.3), we have

$$
\begin{gathered}
\frac{1}{2^{g-2}(g-2) !} \int_{\Sigma^{g-2}} \prod_{k}\left|d w_{k}\right|^{2} \operatorname{det} \omega\left(z_{1}, z_{2}, w_{1}, \ldots, w_{g-2}\right) \operatorname{det} \bar{\omega}\left(z_{3}, z_{4}, w_{1}, \ldots, w_{g-2}\right)= \\
\sum_{\substack{i<j \\
m<n}}(-1)^{i+j+m+n}\left[\omega_{i}\left(z_{1}\right) \omega_{j}\left(z_{2}\right)-\omega_{j}\left(z_{1}\right) \omega_{i}\left(z_{2}\right)\right] \operatorname{det}_{\substack{k \neq i, j \\
l \neq m, n}} \operatorname{Im} \Omega_{k l}\left[\bar{\omega}_{m}\left(z_{3}\right) \bar{\omega}_{n}\left(z_{4}\right)-\bar{\omega}_{n}\left(z_{3}\right) \bar{\omega}_{m}\left(z_{4}\right)\right] .
\end{gathered}
$$

This shows that partial integration on the variables of products of determinants provides a method to construct linear combinations of $\omega_{i}\left(z_{1}\right) \omega_{j}\left(z_{2}\right) \bar{\omega}_{k}\left(z_{3}\right) \bar{\omega}_{l}\left(z_{4}\right)$, with the requested properties under modular transformations.

\subsection{The integration over $\Sigma^{2 g}$}

In [3], the amplitudes calculated using the genus two formula were compared with the corresponding terms in the type IIB low energy effective action. In particular, the two-loop contribution to the $D^{4} R^{4}$ term, predicted by $S L(2, \mathbb{Z})$ duality [21], was obtained by setting the momenta $k_{i}=0$ in the exponential of the integrand of the 4-points amplitude formula. More precisely, in [3] the identity

$$
\Delta\left(z_{1}, z_{2}\right) \Delta\left(z_{3}, z_{4}\right)+\Delta\left(z_{1}, z_{4}\right) \Delta\left(z_{2}, z_{3}\right)+\Delta\left(z_{1}, z_{3}\right) \Delta\left(z_{4}, z_{2}\right)=0
$$

where $\Delta\left(z_{i}, z_{j}\right):=\omega_{1}\left(z_{i}\right) \omega_{2}\left(z_{j}\right)-\omega_{2}\left(z_{i}\right) \omega_{1}\left(z_{j}\right)$, implied

$\int_{\Sigma^{4}}\left|\mathcal{Y}_{S}\right|^{2}=\int_{\Sigma^{4}}\left|s \Delta\left(z_{1}, z_{4}\right) \Delta\left(z_{2}, z_{3}\right)-t \Delta\left(z_{1}, z_{2}\right) \Delta\left(z_{3}, z_{4}\right)\right|^{2}=32\left(s^{2}+t^{2}+u^{2}\right)(\operatorname{det} \operatorname{Im} \Omega)^{2}$

We note that, at generic $g$, the symmetries of the function $\mathcal{Y}_{S}$ under the exchange $\left(z_{i}, k_{i}\right) \leftrightarrow\left(z_{j}, k_{j}\right)$ and the relation $s+t+u=0$ constrain $\int_{\Sigma^{2 g}}\left|\mathcal{Y}_{S}\right|^{2}$ to be proportional to $\left(s^{2}+t^{2}+u^{2}\right) \operatorname{det}(\operatorname{Im} \Omega)^{2}$. The term $s^{2}+t^{2}+u^{2}$ is the unique homogeneous polynomial quadratic in the Mandelstam variables which is symmetric under the exchanges $u \leftrightarrow s$, $t \leftrightarrow s, u \leftrightarrow t$. Furthermore, since $\mathcal{Y}_{S}$ is a product of two determinants made of the canonical basis of holomorphic one-differentials, modular invariance implies that this integral must be proportional to $(\operatorname{det} \operatorname{Im} \Omega)^{2}$.

In the following we will show that, by a suitable choice of the coefficients $C(\rho)$ defining $\mathcal{H}_{i j, k l}$ in (3.1), the relation (3.6) can be generalized to

$$
\mathcal{H}_{12,34}+\mathcal{H}_{14,23}+\mathcal{H}_{13,42}=0
$$


The above remarks suggest that with such a choice of the coefficients $C(\rho)$, a generalization of (3.7) holds also at higher genus. It is instructive to explicitly perform the calculations that will also provide the exact numerical factors.

In the remaining of this section we will consider the following steps.

1. First we will derive a formula that will allow us to easily compute

$$
\begin{aligned}
\mathcal{L}_{1} & :=\int_{\Sigma^{2 g}}\left|\mathcal{H}_{12,34}\right|^{2}, \\
\mathcal{L}_{2} & :=\int_{\Sigma^{2 g}} \mathcal{H}_{14,23} \overline{\mathcal{H}}_{12,34} .
\end{aligned}
$$

2. Next we will show that the condition (3.8) is equivalent to $\mathcal{L}_{1}=-2 \mathcal{L}_{2}$ and this will be used to find a class of coefficients $C(\rho)$ satisfying (3.8).

3. Finally, for this class of coefficients, we will compute $\int_{\Sigma^{2 g}}\left|\mathcal{Y}_{S}\right|^{2}$.

\subsection{Combinatorics of determinants}

Let $\tau$ be a permutation of the set $\{1,2, \ldots, 2 g\}$. We define the following sets

$$
\begin{aligned}
D_{1} & :=\{1,2, \ldots, g\} \cap \tau^{-1}(\{1,2, \ldots, g\}), \\
D_{2} & :=\{g+1, g+2, \ldots, 2 g\} \cap \tau^{-1}(\{g+1, g+2, \ldots, 2 g\}), \\
I_{1} & :=\{g+1, g+2, \ldots, 2 g\} \cap \tau^{-1}(\{1,2, \ldots, g\}), \\
I_{2} & :=\{1,2, \ldots, g\} \cap \tau^{-1}(\{g+1, g+2, \ldots, 2 g\}) .
\end{aligned}
$$

Note that $\operatorname{Card} D_{1}=\operatorname{Card} D_{2}=g-m$, where $m:=\operatorname{Card} I_{1}=\operatorname{Card} I_{2}$.

Theorem 3. Let $\left\{x_{1}, x_{2}, \ldots, x_{2 g}\right\}$ be a set of $2 g$ points in $\Sigma$, $\tau$ a permutation of $\{1,2, \ldots, 2 g\}$ and $D_{1}, D_{2}, I_{1}, I_{2}$ and $m$ as above. Then

$$
\begin{aligned}
P(\tau) & :=\int_{\Sigma^{2 g}} X\left(x_{1 \rightarrow g}\right) X\left(x_{g+1 \rightarrow 2 g}\right) \bar{X}\left(x_{\tau^{-1}(1 \rightarrow g)}\right) \bar{X}\left(x_{\tau^{-1}(g+1 \rightarrow 2 g)}\right)= \\
& = \pm g !(g-m) ! m !(\operatorname{det} A)^{2}
\end{aligned}
$$

where $A:=2 \operatorname{Im} \Omega$, and the sign depends on the permutation $\tau$. 
Proof: We have

$$
\begin{array}{r}
P(\tau)=\int_{\Sigma^{2 g}} \epsilon(i) \epsilon(j) \epsilon(k) \epsilon(l) \omega_{i_{1}}\left(x_{1}\right) \ldots \omega_{i_{g}}\left(x_{g}\right) \omega_{j_{1}}\left(x_{g+1}\right) \ldots \omega_{j_{g}}\left(x_{2 g}\right) . \\
\cdot \prod_{r \in D_{1}} \bar{\omega}_{k_{\tau(r)}}\left(x_{r}\right) \prod_{s \in I_{1}} \bar{\omega}_{k_{\tau(s)}}\left(x_{s}\right) \prod_{t \in I_{2}} \bar{\omega}_{l_{\tau(t)-g}}\left(x_{t}\right) \prod_{u \in D_{2}} \bar{\omega}_{l_{\tau(u)-g}}\left(x_{u}\right),
\end{array}
$$

where $\epsilon(i):=\epsilon^{i_{1} i_{2} \ldots i_{g}}$. Performing the integration, we obtain

$$
P(\tau)=\epsilon(i) \epsilon(j) F(i, j),
$$

where

$$
F(i, j):=\epsilon(k) \epsilon(l) \prod_{r \in D_{1}} A_{i_{r} k_{\tau(r)}} \prod_{s \in I_{1}} A_{j_{s-g} k_{\tau(s)}} \prod_{t \in I_{2}} A_{i_{t} l_{\tau(t)-g}} \prod_{u \in D_{2}} A_{j_{u-g} l_{\tau(u)-g}} .
$$

The indices $i$ 's and $j$ 's are just permutations of the set $\{1,2, \ldots, g\}$, because of the factors $\epsilon(i) \epsilon(j)$ in (3.9). This means that, for each $s \in I_{1}, j_{s-g}$ must be equal to one and only one $i_{t}, t \in D_{1} \cup I_{2}$. If $i_{\hat{r}}=j_{\hat{s}-g}$ for some $\hat{r} \in D_{1}$ and $\hat{s} \in I_{1}$, we can write

$$
F(i, j)=\epsilon(k) \epsilon(l) A_{i_{\hat{r}} k_{\tau(\hat{r})}} A_{j_{\hat{s}-g} k_{\tau(s)}} \cdot(\text { other terms), }
$$

where the terms in the brackets do not depend on $k_{\tau(\hat{r})}$ and $k_{\tau(\hat{s})}$. In this case, $F(i, j)$ is both symmetric and skew-symmetric in $k_{\tau(\hat{r})}$ and $k_{\tau(\hat{s})}$, so it must vanish identically. Thus, a necessary condition for $F(i, j)$ to be non-vanishing is the existence of a bijective map

$$
\eta: I_{2} \rightarrow I_{1}
$$

such that $j_{\eta(t)-g}=i_{t}$ for all $t \in I_{2}$. In this case, we can write

$$
F(i, j)=\epsilon(k) \epsilon(l) \prod_{r \in D_{1}} A_{i_{r} k_{\tau(r)}} \prod_{t \in I_{2}} A_{i_{t} k_{\tau \circ \eta(t)}} \prod_{s \in I_{1}} A_{j_{s-g} l_{\left(\tau \circ \eta^{-1}\right)(s)-g}} \prod_{u \in D_{2}} A_{j_{u-g} l_{\tau(u)-g}} .
$$

Let us define

$$
\lambda(r):= \begin{cases}\tau(r) & \text { if } r \in D_{1} \\ \tau \circ \eta(r) & \text { if } r \in I_{2}\end{cases}
$$

and

$$
\mu(t):= \begin{cases}\tau(t) & \text { if } t \in D_{2}, \\ \tau \circ \eta^{-1}(t) & \text { if } t \in I_{1},\end{cases}
$$

which are permutations of the sets $\{1, \ldots, g\}$ and $\{g+1, \ldots, 2 g\}$, respectively. We have

$$
\begin{aligned}
F(i, j) & =\epsilon(k) \epsilon(l) \prod_{r=1}^{g} A_{i_{r} k_{\lambda(r)}} \prod_{s=1}^{g} A_{j_{s} l_{\mu(s+g)-g}}= \\
& =\epsilon(k) \epsilon(l) \operatorname{sgn}(\lambda) \operatorname{sgn}(\mu) \prod_{r=1}^{g} A_{i_{r} k_{r}} \prod_{s=1}^{g} A_{j_{s} l_{s}}=\epsilon(i) \epsilon(j) \operatorname{sgn}(\lambda) \operatorname{sgn}(\mu)(\operatorname{det} A)^{2}
\end{aligned}
$$


where we have relabeled $k_{\lambda(r)} \rightarrow k_{r}$ and $l_{\mu(s+g)-g} \rightarrow l_{s}$. The term $\operatorname{sgn}(\lambda) \operatorname{sgn}(\mu)$ comes from the rearrangement of the $k$ 's and l's in $\epsilon(k) \epsilon(l)$, and we used the identity

$$
\epsilon(k) \prod_{r=1}^{g} A_{i_{r} k_{r}}=\epsilon(i) \operatorname{det} A
$$

Note that, for a fixed $\tau$, there are, in general, several different choices for the map $\eta$. If we change $\eta \rightarrow \eta^{\prime}$, we have

$$
\begin{aligned}
& \operatorname{sgn}\left(\lambda^{\prime}\right)=\operatorname{sgn}\left(\eta^{\prime} \circ \eta^{-1}\right) \operatorname{sgn}(\lambda), \\
& \operatorname{sgn}\left(\mu^{\prime}\right)=\operatorname{sgn}\left(\eta^{\prime-1} \circ \eta\right) \operatorname{sgn}(\mu),
\end{aligned}
$$

and then

$$
\operatorname{sgn}\left(\lambda^{\prime}\right) \operatorname{sgn}\left(\mu^{\prime}\right)=\operatorname{sgn}(\lambda) \operatorname{sgn}(\mu) .
$$

Therefore, the product $\operatorname{sgn}(\lambda) \operatorname{sgn}(\mu)$ depends only on $\tau$ and not on $\eta$. We find that if $F(i, j)$ is nonzero, then it depends on the $i$ 's and $j$ 's only through the product $\epsilon(i) \epsilon(j)$.

We now insert (3.12) into (3.9) and sum over all the sets of indices $i$ and $j$ for which $F(i, j)$ is not null. We can arbitrarily choose the values of $i_{1}, \ldots, i_{g}$; there are $g$ ! different possibilities. We must choose the $j$ 's in such a way that there exists a function $\eta$ as above; this is a constraint on the values of $j_{s-g}, s \in I_{1}$. There are exactly $m$ ! different bijections $\eta$ from $I_{2}$ to $I_{1}$. Finally we can choose the values of the remaining $g-m$ indices $j_{u}$, for $u \in D_{2}$, that gives a factor $(g-m)$ !. We obtain

$$
P(\tau)=\operatorname{sgn}(\lambda) \operatorname{sgn}(\mu) g !(g-m) ! m !(\operatorname{det} A)^{2} .
$$

\subsection{Evaluation of $\mathcal{L}_{1}$ and $\mathcal{L}_{2}$.}

We now use theorem 3 to compute $\mathcal{L}_{1}$ and $\mathcal{L}_{2}$. These terms split into two contributions

$$
\mathcal{L}_{i}=2\left(\mathcal{L}_{i}^{A}+\mathcal{L}_{i}^{B}\right)
$$

where

$$
\mathcal{L}_{i}^{A, B}=\sum_{\rho, \sigma \in B_{2 g-4}^{\prime}} C(\rho) \bar{C}(\sigma) \int_{\Sigma^{2 g}} L_{i}^{A, B}
$$

and

$$
\begin{aligned}
& L_{1}^{A}:=X_{12}\left(w_{\rho(1 \rightarrow g-2)}\right) X_{34}\left(w_{\rho(g-1 \rightarrow 2 g-4)}\right) \bar{X}_{12}\left(w_{\sigma(1 \rightarrow g-2)}\right) \bar{X}_{34}\left(w_{\sigma(g-1 \rightarrow 2 g-4)}\right), \\
& L_{1}^{B}:=X_{12}\left(w_{\rho(1 \rightarrow g-2)}\right) X_{34}\left(w_{\rho(g-1 \rightarrow 2 g-4)}\right) \bar{X}_{34}\left(w_{\sigma(1 \rightarrow g-2)}\right) \bar{X}_{12}\left(w_{\sigma(g-1 \rightarrow 2 g-4)}\right), \\
& L_{2}^{A}:=X_{14}\left(w_{\rho(1 \rightarrow g-2)}\right) X_{23}\left(w_{\rho(g-1 \rightarrow 2 g-4)}\right) \bar{X}_{12}\left(w_{\sigma(1 \rightarrow g-2)}\right) \bar{X}_{34}\left(w_{\sigma(g-1 \rightarrow 2 g-4)}\right), \\
& L_{2}^{B}:=X_{14}\left(w_{\rho(1 \rightarrow g-2)}\right) X_{23}\left(w_{\rho(g-1 \rightarrow 2 g-4)}\right) \bar{X}_{34}\left(w_{\sigma(1 \rightarrow g-2)}\right) \bar{X}_{12}\left(w_{\sigma(g-1 \rightarrow 2 g-4)}\right) .
\end{aligned}
$$


Let us consider $\mathcal{L}_{1}^{A}$. By the change of variables $w_{\rho(i)} \rightarrow w_{i}$ we can replace $L_{1}^{A}$ in (3.13) by

$$
X_{12}\left(w_{1 \rightarrow g-2}\right) X_{34}\left(w_{g-1 \rightarrow 2 g-4}\right) \bar{X}_{12}\left(w_{\rho^{-1} \circ \sigma(1 \rightarrow g-2)}\right) \bar{X}_{34}\left(w_{\rho^{-1} \circ \sigma(g-1 \rightarrow 2 g-4)}\right) .
$$

We define the variables $\left\{x_{i}\right\}, i=1, \ldots, 2 g$ as

$$
\begin{array}{ll}
x_{1}:=z_{1}, & x_{g+1}:=z_{3} \\
x_{2}:=z_{2}, & x_{g+2}:=z_{4} \\
x_{i}:=w_{i-2}, \quad i=3, \ldots, g, & x_{i}:=w_{i-4}, \quad i=g+3, \ldots, 2 g
\end{array}
$$

Then, we have

$$
\mathcal{L}_{1}^{A}=\sum_{\rho, \sigma \in B_{2 g-4}^{\prime}} C(\rho) \bar{C}(\sigma) P(\tau),
$$

where $P(\tau)$ is the expression in the previous theorem and $\tau$ is the permutation

$$
\begin{array}{ll}
\tau(1)=1, & \tau(g+1)=g+1, \\
\tau(2)=2, & \tau(g+2)=g+2, \\
\tau(i)=\sigma^{-1} \circ \rho(i-2), \quad i=3, \ldots, g, & \tau(i)=\sigma^{-1} \circ \rho(i-4), \quad i=g+3, \ldots, 2 g .
\end{array}
$$

Let us define

$$
n:=\operatorname{Card}[\rho(\{1,2, \ldots, g-2\}) \cap \sigma(\{g-1, g, \ldots, 2 g-4\})],
$$

and notice that

$$
n=\operatorname{Card}\left[\{3,4, \ldots, g\} \cap \tau^{-1}(\{g+3, \ldots, 2 g\})\right] .
$$

Since $\{1,2\} \subset D_{1}$, and $\tau^{-1}(\{g+1, g+2\}) \subset D_{2}$, the above intersection corresponds to the set $I_{2}$. Then, by theorem 3 with $m=n$ we obtain

$$
\mathcal{L}_{1}^{A}=\sum_{n=0}^{g-3} F_{n} g !(g-n) ! n !(\operatorname{det} A)^{2}
$$

where

$$
F_{n}=\sum_{(\rho, \sigma) \in B_{2 g-4}^{\prime}(n)} C(\rho) \bar{C}(\sigma) S(\rho, \sigma) .
$$

Here the sum is over all the pairs $(\rho, \sigma) \in B_{2 g-4}^{\prime}(n) \subset B_{2 g-4}^{\prime} \times B_{2 g-4}^{\prime}$, such that the intersection defined in (3.15) has $n$ elements, and

$$
S(\rho, \sigma)=\operatorname{sgn}(\lambda) \operatorname{sgn}(\mu)
$$

where $\lambda$ and $\mu$ are defined in (3.10) and (3.11), with $\eta$ an arbitrary bijective function from $I_{2}$ into $I_{1}$. Note that $\lambda(1)=1, \lambda(2)=2, \mu(g+1)=g+1$ and $\mu(g+2)=g+2$. 
A similar calculation can be performed for $\mathcal{L}_{1}^{B}$. We use the same change of variables for the $\left\{w_{i}\right\}$ and the same definition for the variables $\left\{x_{i}\right\}$. In this case, the permutation $\tau$ is given by

$$
\begin{array}{ll}
\tau(1)=g+1, & \tau(g+1)=1, \\
\tau(2)=g+2, & \tau(g+2)=2,
\end{array}
$$

while the other entries are the same as in the previous case. We define $n$ as in (3.15). In this case $\{1,2\}=\tau^{-1}(\{g+1, g+2\}) \subset I_{2}$, so that, we can apply theorem 3 with $m=n+2$ to get

$$
\mathcal{L}_{1}^{B}=\sum_{n=0}^{g-3} F_{n} g !(g-n-2) !(n+2) !(\operatorname{det} A)^{2},
$$

where $F_{n}$ is defined in (3.16). No extra minus sign comes from the product $\operatorname{sgn}(\lambda) \operatorname{sgn}(\mu)$. To see this, notice that we can choose an arbitrary $\eta$ in the definition, since this product does not depend on this choice. We set $\eta(1)=g+1$ and $\eta(2)=g+2$, and obtain $\lambda(1)=1$, $\lambda(2)=2, \mu(g+1)=g+1$ and $\mu(g+2)=g+2$. These are exactly the same relations as for $\mathcal{L}_{1}^{A}$, and then we get the same overall sign.

Let us now consider $\mathcal{L}_{2}^{A}$ and $\mathcal{L}_{2}^{B}$. In the case of $\mathcal{L}_{2}^{A}$ the permutation $\tau$ is defined by

$$
\begin{array}{lll}
\tau(1)=1, & \tau(g+1)=2, \\
\tau(2)=g+2, & \tau(g+2)=g+1,
\end{array}
$$

while in the case of $\mathcal{L}_{2}^{B}$ we have

$$
\begin{array}{ll}
\tau(1)=g+1, & \tau(g+1)=g+2, \\
\tau(2)=2, & \tau(g+2)=1 .
\end{array}
$$

We have $\{1\} \subset D_{1},\{2\} \subset I_{2},\{g+1\} \subset I_{1}$ and $\{g+2\} \subset D_{2}$ in the $\mathcal{L}_{2}^{A}$ case, while $\{1\} \subset I_{2}$, $\{2\} \subset D_{1},\{g+1\} \subset D_{2}$ and $\{g+2\} \subset I_{1}$ in the $\mathcal{L}_{2}^{B}$ case. Thus, we set $m=n+1$ and obtain

$$
\mathcal{L}_{2}^{A}=\mathcal{L}_{2}^{B}=-\sum_{n=0}^{g-3} F_{n} g !(g-n-1) !(n+1) !(\operatorname{det} A)^{2} .
$$

The minus sign arises because of the product $\operatorname{sgn}(\lambda) \operatorname{sgn}(\mu)$. To see this in the $\mathcal{L}_{2}^{A}$ case, we choose $\eta(2)=g+1$ and obtain $\lambda(1)=1, \lambda(2)=2, \mu(g+1)=g+2$ and $\mu(g+2)=g+1$. The interchange $g+1 \leftrightarrow g+2$ in the $\mu$ permutation gives the minus sign. A similar computation can be performed for $\mathcal{L}_{1}^{B}$.

Summarizing, we have

$$
\begin{aligned}
& \mathcal{L}_{1}=2 \sum_{n=0}^{g-3} F_{n} g ![(g-n) ! n !+(g-n-2) !(n+2) !] 2^{2 g}(\operatorname{det} \operatorname{Im} \Omega)^{2}, \\
& \mathcal{L}_{2}=-4 \sum_{n=0}^{g-3} F_{n} g !(g-n-1) !(n+1) ! 2^{2 g}(\operatorname{det} \operatorname{Im} \Omega)^{2},
\end{aligned}
$$


where $F_{n}$ is defined in (3.16). We remark that these formulas hold for generic choices of the coefficients $C(\rho)$.

\subsection{A condition on the building blocks}

We claimed that the condition (3.8) is equivalent to the following constraint

$$
\mathcal{L}_{1}=-2 \mathcal{L}_{2}
$$

that is

$$
\sum_{n=0}^{g-3} F_{n}[(g-n) ! n !+(g-n-2) !(n+2) !-4(g-n-1) !(n+1) !]=0 .
$$

Actually, we trivially have

$$
\begin{aligned}
& 3 \mathcal{L}_{1}=\int_{\Sigma^{2 g}}\left|\mathcal{H}_{12,34}\right|^{2}+\int_{\Sigma^{2 g}}\left|\mathcal{H}_{13,42}\right|^{2}+\int_{\Sigma^{2 g}}\left|\mathcal{H}_{14,23}\right|^{2} \\
& 3 \mathcal{L}_{2}=\int_{\Sigma^{2 g}} \mathcal{H}_{12,34} \overline{\mathcal{H}}_{13,42}+\int_{\Sigma^{2 g}} \mathcal{H}_{12,34} \overline{\mathcal{H}}_{14,23}+\int_{\Sigma^{2 g}} \mathcal{H}_{13,42} \overline{\mathcal{H}}_{14,23}
\end{aligned}
$$

where, in each sum, the three terms are related each other by suitable changes of variables. Then, the relation $3\left(\mathcal{L}_{1}+2 \mathcal{L}_{2}\right)=0$ reads

$$
\int_{\Sigma^{2 g}}\left|\mathcal{H}_{12,34}+\mathcal{H}_{14,23}+\mathcal{H}_{13,42}\right|^{2}=0
$$

that is Eq.(3.8). On the other hand, if (3.8) holds, then

$$
\int_{\Sigma^{2 g}}\left|\mathcal{Y}_{S}\right|^{2}=\int_{\Sigma^{2 g}}\left|s \mathcal{H}_{14,23}-t \mathcal{H}_{12,34}\right|^{2}=\left(s^{2}+t^{2}\right) \mathcal{L}_{1}-2 s t \mathcal{L}_{2}
$$

Since $\int_{\Sigma^{2 g}}\left|\mathcal{Y}_{S}\right|^{2}$ is proportional to $s^{2}+t^{2}+u^{2}=2\left(s^{2}+t^{2}+s t\right)$, this implies (3.17). Note that we can use the formulas for $\mathcal{L}_{1}$ and $\mathcal{L}_{2}$ to evaluate $\int_{\Sigma^{2 g}}\left|\mathcal{Y}_{S}\right|^{2}$.

In the following we will consider the general solutions for the constraints (3.18) in the cases $g=3$ and $g=4$ and then we will propose a simple solution for general $g$. In this case, we will also compute the integral $\int_{\Sigma^{2 g}}\left|\mathcal{Y}_{S}\right|^{2}$.

In the case $g=3$, there are two additional points $w_{1}$ and $w_{2}$. There is only a permutation in $B_{2 g-4}^{\prime}$; its coefficient $C$ is just a normalization of $\mathcal{H}_{i j, k l}$. We have $F_{0}=|C|^{2}$ and the condition (3.18) is an identity.

For $g=4$, we have four additional points $w_{1}, \ldots, w_{4}$ and three different permutations in $B_{2 g-4}^{\prime}$

$$
\rho_{1}=(1,2,3,4), \quad \rho_{2}=(1,4,3,2), \quad \rho_{3}=(1,3,2,4)
$$


with coefficients $C_{1}, C_{2}, C_{3}$, respectively. Computing the signs $S\left(\rho_{i}, \rho_{j}\right), i, j=1,2,3$, we find

$$
\begin{aligned}
& F_{0}=\left|C_{1}\right|^{2}+\left|C_{2}\right|^{2}+\left|C_{3}\right|^{2}, \\
& F_{1}=2 \operatorname{Re}\left(C_{1} \bar{C}_{2}-C_{2} \bar{C}_{3}+C_{3} \bar{C}_{1}\right),
\end{aligned}
$$

and the condition (

$$
\left|C_{1}-C_{2}-C_{3}\right|^{2}=0
$$

Thus, we have infinite real or complex solutions for the coefficients $C_{1}, C_{2}, C_{3}$.

For general $g$, there always exists a rather simple solution to (3.18). Let us denote by $S_{2 g-4}^{\prime} \subset B_{2 g-4}^{\prime}$ the set of all permutations $\rho$ such that

$$
\rho(i)= \begin{cases}1 & i=1 \\ i \text { or } i+g-1 & i=2, \ldots, g-2 \\ g-1 & i=g-1 \\ i \text { or } i-g+1 & i=g, \ldots, 2 g-4\end{cases}
$$

At genus $g$, this set has $2^{g-3}$ elements. Then, set

$$
C(\rho)= \begin{cases}1 & \rho \in S_{2 g-4}^{\prime}, \\ 0 & \text { otherwise }\end{cases}
$$

where we absorbed a possible overall constant in $B_{g}$ in (1.2). In the following we show that this is a solution to (3.18).

Fix a pair $(\rho, \sigma) \in S_{2 g-4}^{\prime}$, and define $\tau$ as in (3.14). Each element $r \in I_{2}$ is given by $r=\tau^{-1}(r+g-1)$. Thus, we can choose $\eta(r)=r+g-1$, and we have $\lambda=\mu=\mathrm{id}$. This means that $S(\rho, \sigma)=+1$ for all $\rho, \sigma \in S_{2 g-4}^{\prime}$.

Let us now compute $F_{n}$. Since $C(\rho) \bar{C}(\sigma) S(\rho, \sigma)=1$ for all $\rho, \sigma \in S_{2 g-4}^{\prime}$, it follows that $F_{n}$ is just the number of pairs $(\rho, \sigma)$ satisfying (3.15). We can choose $\rho$ arbitrarily; the permutations $\sigma$ satisfying (3.15) are in one to one correspondence with the ways of choosing $n$ elements in the set $\{2, \ldots, g-2\}$. Thus

$$
F_{n}=2^{g-3} \frac{(g-3) !}{(g-n-3) ! n !},
$$

and by (3.18), we obtain the condition

$$
\sum_{n=0}^{g-3}(g-n-2)[(g-n)(g-n-1)+(n+1)(n+2)-4(g-n-1)(n+1)]=0 .
$$


Now observe that the identity

$$
\sum_{n=0}^{g-3}(g-n-2)(n+2)(n+1)=\sum_{n=0}^{g-3}(n+1)(g-n-1)(g-n-2),
$$

which follows by a redefinition $n \rightarrow g-n-3$, also implies

$$
\sum_{n=0}^{g-3}(g-n-2)[(g-n)(g-n-1)+(n+1)(n+2)]=(g+1) \sum_{n=0}^{g-3}(g-n-1)(g-n-2),
$$

and

$$
4 \sum_{n=0}^{g-3}(n+1)(g-n-1)(g-n-2)=2(g+1) \sum_{n=0}^{g-3}(n+1)(g-n-2) .
$$

Then, (3.20) reduces to

$$
\sum_{n=0}^{g-3}(g-3 n-3)(g-n-2)=0
$$

which is an identity, as one can verify using

$$
\sum_{n=0}^{g-3} n^{2}=\frac{1}{6}(g-2)\left[2(g-2)^{2}-3(g-3)-2\right] .
$$

Thus, we have found a simple form for the building block

$\mathcal{H}_{i j, k l}=\sum_{\rho \in S_{2 g-4}^{\prime}}\left[X_{i j}\left(w_{\rho(1 \rightarrow g-2)}\right) X_{k l}\left(w_{\rho(g-1 \rightarrow 2 g-4)}\right)+X_{k l}\left(w_{\rho(1 \rightarrow g-2)}\right) X_{i j}\left(w_{\rho(g-1 \rightarrow 2 g-4)}\right)\right]$,

where $S_{2 g-4}^{\prime}$ is the subgroup of the permutations $\rho$ satisfying (3.19). By (3.17) and the kinematic relation $s+t+u=0$, we have

$$
\int_{\Sigma^{2 g}}\left|\mathcal{Y}_{S}\right|^{2}=-\left(s^{2}+t^{2}+u^{2}\right) \mathcal{L}_{2}
$$

Eq.(3.23) gives

$$
-\mathcal{L}_{2}=2^{3 g-1} g !(g-3) ! \sum_{n=0}^{g-3}(g-n-1)(g-n-2)(n+1)(\operatorname{det} \operatorname{Im} \Omega)^{2},
$$

and by (3.21) and (3.22), we get

$$
\int_{\Sigma^{2 g}}\left|\mathcal{Y}_{S}\right|^{2}=\frac{2^{3 g-3}}{3}(g+1) !(g-2) !\left(g^{2}-7 g+18\right)\left(s^{2}+t^{2}+u^{2}\right)(\operatorname{det} \operatorname{Im} \Omega)^{2}
$$




\section{Conclusions.}

The 2-loop 4-point superstring amplitude, in the slice independent formulation [1], shows an unexpectedly simple shape and suggests some possible generalization to higher genus. A natural conjecture is that the measure on the moduli space is (the restriction of) the modular invariant measure on the Siegel upper half-space derived in [20]. Furthermore, we argued that the basic building block generalizing the terms $\Delta\left(z_{i}, z_{j}\right) \Delta\left(z_{k}, z_{l}\right)$ in the genus 2 formula, is a sum of terms made of product of 2 determinants of holomorphic abelian differentials. So that, we are naturally lead to the following formula

$$
A_{4}^{g-\text { loop }}=B_{g} \int_{\mathcal{M}_{g}}|\operatorname{det} g|^{1 / 2} \mathrm{~d} \Xi_{1} \wedge \ldots \wedge \mathrm{d} \bar{\Xi}_{3 g-3} \mathcal{F}_{g}\left(k_{i}\right)
$$

In the case of non-hyperelliptic Riemann surfaces of genus 3 we have

$$
A_{4}^{3-\text { loop }}=B_{3} \int_{\mathcal{M}_{3}} \frac{\left|\bigwedge_{i \leq j}^{3} \mathrm{~d} \Omega_{i j}\right|^{2}}{(\operatorname{det} \operatorname{Im} \Omega)^{4}} \int_{\Sigma^{6}} \frac{\left|\mathcal{Y}_{S}\right|^{2}}{(\operatorname{det} \operatorname{Im} \Omega)^{2}} e^{-\sum_{i<j} k_{i} \cdot k_{j} G\left(z_{i}, z_{j}\right)}
$$

where, since there are only two extra points, there is only a possible choice for $\mathcal{H}_{i j, k l}$, namely

$$
\mathcal{H}_{i j, k l}=\operatorname{det} \omega\left(z_{i}, z_{j}, w_{1}\right) \operatorname{det} \omega\left(z_{k}, z_{l}, w_{2}\right)+\operatorname{det} \omega\left(z_{i}, z_{j}, w_{2}\right) \operatorname{det} \omega\left(z_{k}, z_{l}, w_{1}\right)
$$

In the case of genus $g \geq 4$ the definition of $\mathcal{H}_{i j, k l}$ requires choosing a set of permutations over the $2 g-4$ points $\left\{w_{k}\right\}$ and their relative coefficients. The simplest choice is

$$
\mathcal{H}_{12,34}=X_{12}\left(w_{1 \rightarrow g-2}\right) X_{34}\left(w_{g-1 \rightarrow 2 g-4}\right)+X_{34}\left(w_{1 \rightarrow g-2}\right) X_{12}\left(w_{g-1 \rightarrow 2 g-4}\right) .
$$

In the paper we considered the simplest generalization of (1.1). However, more general formulas for the building blocks $\mathcal{H}_{i j, k l}$ can be constructed in terms of products of 4 determinants like det $\omega_{i}\left(x_{j}\right)$, times suitable functions of the kinematical variables. In the four matrices $\omega_{i}\left(x_{j}\right), j=1, \ldots, 4 g$ each point $x_{j}$ corresponds either to one of the insertion points $z_{1}, \ldots, z_{4}$, or to one of the $4 g-4$ additional points $\left\{w_{i}\right\}$, to be eventually integrated away. The integration may give factors that cancel some power in the overall $(\operatorname{det} \operatorname{Im} \Omega)^{4}$ in the denominator inserted to balance the modular transformations of the four determinants and their complex conjugated. Thus, the basic step in defining such building blocks is the distribution of the points $z_{1}, \ldots, z_{4}$ and $\left\{w_{i}\right\}$ in such matrices. There are several different ways to implement such a distribution. In particular, with respect to the position of the 
points $z_{1}, \ldots, z_{4}$, in the determinants, there are five possibilities

$$
\begin{aligned}
& \operatorname{det} \omega\left(z_{i}, w \ldots\right) \operatorname{det} \omega\left(z_{j}, w \ldots\right) \operatorname{det} \omega\left(z_{k}, w \ldots\right) \operatorname{det} \omega\left(z_{l}, w \ldots\right), \\
& \operatorname{det} \omega\left(z_{i}, z_{j}, w \ldots\right) \operatorname{det} \omega\left(z_{k}, w \ldots\right) \operatorname{det} \omega\left(z_{l}, w \ldots\right) \operatorname{det} \omega(w \ldots), \\
& \operatorname{det} \omega\left(z_{i}, z_{j}, \ldots \ldots\right) \operatorname{det} \omega\left(z_{k}, z_{l}, w \ldots\right) \operatorname{det} \omega(w \ldots) \operatorname{det} \omega(w \ldots), \\
& \operatorname{det} \omega\left(z_{i}, z_{j}, z_{k}, w \ldots\right) \operatorname{det} \omega\left(z_{l}, w \ldots\right) \operatorname{det} \omega(w \ldots) \operatorname{det} \omega(w \ldots), \\
& \operatorname{det} \omega\left(z_{i}, z_{j}, z_{k}, z_{l}, w \ldots\right) \operatorname{det} \omega(w \ldots) \operatorname{det} \omega(w \ldots) \operatorname{det} \omega(w \ldots),
\end{aligned}
$$

where $w \ldots$ is a permutation of a subset of suitable cardinality of $\left\{w_{i}\right\}$. Note that the last two combinations of determinants may appear only at $g \geq 3$ and $g \geq 4$ respectively. In the case of determinants which do not contain any insertion point $z_{i}$, the integration over the additional points leads to overall factors $\operatorname{det} \operatorname{Im} \Omega$ reducing the power of $(\operatorname{det} \operatorname{Im} \Omega)^{4}$ in the denominator.

In some cases, this mechanism leads to formulas involving the product of two determinants, as considered in this paper. However, in principle, there could be some interesting generalizations which require combinatorial analysis of rapidly growing complexity.

Remarkably, the construction suggests a natural generalization to the $n$ point functions, where the products of $n$ determinants may still play the role of building blocks. On the other hand, at the moment we can hardly derive the exact kinematical factors they are associated to. An extension of our analysis and the symmetry properties of the amplitudes, could, in principle, constrain the possible shape of such functions. We also note that the systematic use of the determinants of holomorphic one-differentials, may lead to control the factorization properties in the various degeneration limits. This would suggest the appearance of an integrable structure related to the cohomology of $\overline{\mathcal{M}}_{g}$.

Acknowledgements. We would like to thank G. Bertoldi, G. Bonelli, L. Mazzucato, Y. Nakayama, P. Pasti, D. Sorokin, M. Tonin, G. Travaglini, P. Vanhove and C.-J. Zhu, for stimulating discussions. Work partially supported by the European Community's Human Potential Programme under contract MRTN-CT-2004-005104 "Constituents, Fundamental Forces and Symmetries of the Universe". 


\section{References}

[1] E. D'Hoker and D. H. Phong, Phys. Lett. B 529, 241 (2002) [arXiv:hep-th/0110247; Nucl. Phys. B 636, 3 (2002) arXiv:hep-th/0110283; Nucl. Phys. B 636, 61 (2002) arXiv:hep-th/0111016; Nucl. Phys. B 639, 129 (2002) arXiv:hep-th/0111040; Nucl. Phys. B 715, 91 (2005) arXiv:hep-th/0501196.

[2] E. D'Hoker and D. H. Phong, Nucl. Phys. B 715, 3 (2005) [arXiv:hep-th/0501197.

[3] E. D'Hoker, M. Gutperle and D. H. Phong, arXiv:hep-th/0503180.

[4] N. Berkovits, arXiv:hep-th/0503197.

[5] N. Berkovits, JHEP 0004, 018 (2000) [arXiv:hep-th/0001035; JHEP 0009, 046 (2000) arXiv:hep-th/0006003; Int. J. Mod. Phys. A 16, 801 (2001) arXiv:hep-th/0008145; JHEP 0108, 026 (2001) [arXiv:hep-th/0104247]; JHEP 0204, 037 (2002) arXiv:hepth/0203248; arXiv:hep-th/0209059; JHEP 0409, 047 (2004) arXiv:hep-th/0406055; Comptes Rendus Physique 6, 185 (2005) arXiv:hep-th/0410079; JHEP 0503, 041 (2005) arXiv:hep-th/0411170.

[6] N. Berkovits and B. C. Vallilo, JHEP 0007, 015 (2000) arXiv:hep-th/0004171. N. Berkovits and O. Chandia, Phys. Lett. B 514, 394 (2001) arXiv:hep-th/0105149; JHEP 0208, 040 (2002) arXiv:hep-th/0204121. N. Berkovits and P. S. Howe, Nucl. Phys. B 635, 75 (2002) arXiv:hep-th/0112160. N. Berkovits and V. Pershin, JHEP 0301, 023 (2003) arXiv:hep-th/0205154. N. Berkovits and N. Seiberg, JHEP 0307, 010 (2003) arXiv:hep-th/0306226. N. Berkovits and D. Z. Marchioro, JHEP

0501, 018 (2005) arXiv:hep-th/0412198. N. Berkovits and N. Nekrasov, arXiv:hepth/0503075.

[7] I. Oda and M. Tonin, Phys. Lett. B 520, 398 (2001) arXiv:hep-th/0109051; Phys. Lett. B 606, 218 (2005) arXiv:hep-th/0409052; arXiv:hep-th/0505277; arXiv:hepth/0506054.

[8] M. Matone, L. Mazzucato, I. Oda, D. Sorokin and M. Tonin, Nucl. Phys. B 639, 182 (2002) arXiv:hep-th/0206104.

[9] P. A. Grassi, G. Policastro, M. Porrati and P. Van Nieuwenhuizen, JHEP 0210, 054 (2002) arXiv:hep-th/0112162]. P. A. Grassi, G. Policastro and P. van Nieuwenhuizen, JHEP 0211, 001 (2002) arXiv:hep-th/0202123; Adv. Theor. Math. Phys. 7, 499 (2003) arXiv:hep-th/0206216; Phys. Lett. B 553, 96 (2003) arXiv:hep-th/0209026; Class. Quant. Grav. 20, S395 (2003) [arXiv:hep-th/0302147]; Nucl. Phys. B 676, 43 (2004) arXiv:hep-th/0307056; arXiv:hep-th/0402122.

[10] L. Anguelova and P. A. Grassi, JHEP 0311, 010 (2003) arXiv:hep-th/0307260. P. A. Grassi and L. Tamassia, JHEP 0407, 071 (2004) arXiv:hep-th/0405072. P. A. Grassi and P. van Nieuwenhuizen, Phys. Lett. B 610, 129 (2005) arXiv:hep th/0408007]. 
[11] B. C. Vallilo, JHEP 0212, 042 (2002) arXiv:hep-th/0210064; JHEP 0403, 037 (2004) arXiv:hep-th/0307018. O. Chandia and B. C. Vallilo, JHEP 0404, 041 (2004) arXiv:hep-th/0401226.

[12] G. Trivedi, Mod. Phys. Lett. A 17, 2239 (2002) [arXiv:hep-th/0205217.

[13] R. Schiappa and N. Wyllard, arXiv:hep-th/0503123.

[14] Y. Aisaka and Y. Kazama, JHEP 0302, 017 (2003) arXiv:hep-th/0212316; JHEP 0308, 047 (2003) arXiv:hep-th/0305221; JHEP 0404, 070 (2004) arXiv:hepth/0404141; arXiv:hep-th/0502208.

[15] L. Cornalba, M. S. Costa and R. Schiappa, arXiv:hep-th/0209164.

[16] P. Mukhopadhyay, arXiv:hep-th/0505157.

[17] R. Iengo and C. J. Zhu, Phys. Lett. B 212, 309 (1988); Phys. Lett. B 212, 313 (1988).

[18] Z. J. Zheng, J. B. Wu and C. J. Zhu, Phys. Lett. B 559, 89 (2003) arXiv:hepth/0212191]; Nucl. Phys. B 663, 79 (2003) [arXiv:hep-th/0212198]; Nucl. Phys. B 663, 95 (2003) arXiv:hep-th/0212219.

[19] C. J. Zhu, arXiv:hep-th/0503001.

[20] M. Matone and R. Volpato, arXiv:math.ag/0506550.

[21] M. B. Green, H. h. Kwon and P. Vanhove, Phys. Rev. D 61, 104010 (2000) arXiv:hepth/9910055]. M. B. Green and P. Vanhove, Phys. Rev. D 61, 104011 (2000) arXiv:hepth/9910056].

[22] E. Arbarello, M. Cornalba, P.A. Griffiths and J. Harris, "Geometry of Algebraic Curves", Vol.1. Berlin, Heidelberg, New-York, Tokyo, 1985.

[23] Y. I. Manin, Phys. Lett. B 172, 184 (1986). A. A. Beilinson and Y. I. Manin, Commun. Math. Phys. 107, 359 (1986).

[24] A. A. Belavin and V. G. Knizhnik, Phys. Lett. B 168, 201 (1986). E. P. Verlinde and H. L. Verlinde, Nucl. Phys. B 288, 357 (1987). E. D'Hoker and D. H. Phong, Rev. Mod. Phys. 60, 917 (1988).

[25] A. Morozov, Phys. Lett. B 184, 171 (1987).

[26] M. Matone, Lett. Math. Phys. 33, 75 (1995) arXiv:hep-th/9310066. 